\title{
Appropriate Use Criteria for Echocardiography in the Era of Value-Based Care: Mission Accomplished or Future Mandates?
}

\author{
lan Hackett ${ }^{1} \cdot$ R. Parker Ward ${ }^{1}$ \\ Published online: 19 June 2020 \\ (C) Springer Science+Business Media, LLC, part of Springer Nature 2020
}

\begin{abstract}
Purpose of Review The purpose of this review is to highlight the past impact and current role of the Appropriate Use Criteria (AUC) for echocardiography in value-based healthcare, and to address future implications in light of the recent mandate from the Centers for Medicare and Medicaid Services to incorporate AUC for other imaging modalities.

Recent Findings Several studies have proven that the AUC effectively stratify the clinical practice of echocardiography as they predict important echo abnormalities and impact optimal patient care. Recent investigations have tested new technologies and demonstrated the feasibility and scalability of the application of the AUC for echocardiography at the point of care.

Summary The AUC for echocardiography has accomplished their core mission, as utilization has moderated over the last decade and mandatory implementation at the point of care for echocardiography remains rare. While a new mandate signals another wave of focus on appropriate utilization, echocardiography stands ready.
\end{abstract}

Keywords Echocardiography $\cdot$ Healthcare policy $\cdot$ Appropriate use $\cdot$ Value-based care

\section{Introduction}

Appropriate Use Criteria (AUC) for a variety for medical procedures and services have been available for over a decade and are now familiar to a majority of healthcare providers. AUC were spawned in an era of unfettered increases in healthcare utilization and healthcare costs, resulting in new scrutiny on the practice of medicine from both governmental and private payers. The development of AUC represented a physician-led response to this changing landscape, with the goal to provide an evidence-based guide for optimal utilization of medical procedures in the delivery of high-quality care. Initial AUC focused on cardiac imaging, targeting utilization growth rates that outpaced other medical services. Echocardiography was a significant contributor to this growth and remains the highest volume cardiac imaging test. The

This article is part of the Topical Collection on Echocardiography

R. Parker Ward

pward@medicine.bsd.uchicago.edu

Ian Hackett

ian.hackett@uchospitals.edu

1 Section of Cardiology, University of Chicago Medicine, $5841 \mathrm{~S}$. Maryland Ave, DCAM Rm 5726, Chicago, IL 60637, USA
AUC for echocardiography have now been refined and applied in a variety of healthcare settings, and in combination with other AUC have accomplished their mission by contributing to a now decade-long correction in the utilization of imaging procedures. However, while AUC are available and incorporated into practice in limited ways for all cardiac imaging modalities, fears of widespread or universal AUC implementation requirements have not materialized. A recent mandate from the Centers for Medicare and Medicaid Services (CMS) for advanced imaging procedures represents the arrival of the long anticipated mandatory AUC implementation requirement. While this mandate does not address echocardiography, it portends a new era of focus on appropriate use of all medical imaging procedures. The purpose of this review is to discuss the past impact, current role, and potential future applications of the Appropriate Use Criteria in the utilization, delivery, and reimbursement of echocardiography in this evolving era of value-based healthcare.

\section{The CMS Mandate for AUC Implementation for Advanced Imaging Services}

A CMS mandate requiring AUC determination for all "advanced imaging procedures" starting January 1, 2021, has 
recently been released. This mandate finally enforces a provision included in the 2014 Protecting Access to Medicare Act (PAMA), and requires providers to consult relevant AUC at the time of test order using a qualified clinical support decision mechanism (CDSM) in order to receive reimbursement from CMS. Advanced imaging procedures are defined as computed tomography $(\mathrm{CT})$, magnetic resonance imaging (MRI), and nuclear medicine studies, including single-photon emission computed tomography (SPECT)/positron emission tomography (PET) myocardial perfusion imaging (MPI), but notably does not include echocardiography procedures.

A CDSM is an interactive electronic tool, usually incorporated in electronic health record (EHR) systems, which provides determination as to whether the order adheres to AUC. Starting January 1, 2021, CDSM-generated AUC determination will be required for any claim for advanced imaging services to be considered. As of January 1, 2020, this program has entered a 1-year testing and education phase, where CDSM-derived AUC determination is expected with all claims, but CMS will not deny payment for those that fail to include or incorrectly report AUC determinations. As currently outlined, even when fully implemented, this program requires inclusion of CDSM-derived AUC reporting, but will not deny payment based on AUC determination. However, practitioners who are considered "outliers" will then be subject to prior authorization procedures. CMS notes that methodology of how "outliers" will be defined and how prior authorization procedures will be implemented "are not yet available" [1].

Of note, at the time of this writing, a coalition of 18 medical societies has sent a letter to the United States Congress requesting that the testing and education phase of this CMS mandate be extended, and that the full implementation start date of January 1, 2021, be suspended, due to anticipated challenges in meeting that deadline for many providers in light of the COVID-19 pandemic.

\section{Why Should Echo Stakeholders Care About a Mandate that Does Not Address Echocardiography?}

As cardiac imaging has become a focal point of intense scrutiny on the potential overuse and increasing expenditures on healthcare procedures and services over the last decade, echocardiography has held a unique role. On the one hand, while data from the pre-AUC era confirmed rapid growth in the utilization of echo procedures, this growth rate paled in comparison with cardiac imaging brethren such as MRI, CT, and SPECT/PET MPI $[2,3]$. This is not surprising given that many of these newer cardiac imaging modalities were experiencing a formative era of evolving technology and expanding indications. However, because echocardiography was and remains the highest volume cardiac imaging test, echo procedures still garnered attention as a modality with perhaps the greatest potential opportunity for absolute cost savings [2]. Despite this, the high volume and widespread availability appears to have insulated echo practice from the most disruptive interventions. For example, many private payers have long since implemented prior authorization programs for advanced modalities, such as cardiac CT, MRI, and nuclear medicine procedures, with the AUC commonly incorporated in the framework of these programs. But similar prior authorization programs remain rare for echocardiography. This is likely due to recognition of how disruptive and expensive this would be for both providers and payers to implement and administer these programs for such a high volume test. Also contributing is the wider range of established indications for echocardiography compared with other modalities, which makes seamless and accurate implementation more daunting. Instead, the only uniform application of AUC for echocardiography to date has been minimal requirements for AUC tracking and reporting of a handful of studies as part of echocardiography laboratory accreditation. According to the Intersocietal Accreditation Commission (IAC) Echocardiography Laboratory Standards from 2018, a "minimum of two cases per quarter must be evaluated for the appropriateness of the test performed" and characterized by the AUC $[4,5]$.

The CMS mandate appears to follow this pattern, focusing for now only on lower volume advanced imaging modalities. However, this may well represent a temporary reprieve. As CDSM programs are refined, become routinely incorporated in EHRs, and providers adapt to online AUC determinations at the time of order, many of the concerns about additionally applying this process to echo practice may dissipate. For all of these reasons, it is prudent that stakeholders take notice and begin to prepare for the potential of similar mandates for the practice of echocardiography.

\section{How Did We Get Here? The Evolving Landscape of Value-Based Care}

The CMS states that the national healthcare expenditure in 2018 is over $\$ 11,000$ per person, with an estimated $30 \%$ of healthcare dollars spent on services that "do not improve patient care" [6]. Similar analysis and conclusions have been widely publicized for two decades and have served as a core premise prompting the ongoing national debate over healthcare reform. Medical diagnostic imaging has been a major target of these reforms, based on a growth rate in utilization and expenditures exceeding other medical services in the early years of the millennium. Between 2000 and 2009, medical diagnostic imaging procedures increased by $85 \%$ [2, 
3]. Echocardiography was not immune to this rapid growth, doubling in volume during this time period.

An additional contributing factor to the focus on medical imaging is hinted at in the phrase "do not improve patient care". A long-standing challenge for medical imaging, particularly cardiac imaging, has been the relative paucity of randomized trials establishing a direct link between performance of a cardiac imaging test and improved patient outcomes. This issue was a focus of a 2016 American College of Cardiology Think Tank and continues to be an important issue today [7]. For example, it is established that a patient with a reduced left ventricular ejection fraction (LVEF) has an increased mortality, and that medications proven to reduce that mortality are available. However, despite the obviously important role echocardiography commonly plays in this scenario, proving an independent association between the performance of an echocardiogram in such a patient and improved outcome is challenging. Make no mistake, there are many investigations, some discussed elsewhere in this review, in which echocardiogram performance is associated with improved outcomes. But the standard of direct independent causality as demonstrated in randomized trials is difficult to achieve and not available for most imaging tests. As a result, when CMS or other governmental decision makers allocate already scant healthcare resources, therapies with proven outcomes ranging from statin prescriptions to hemodialysis become easily justifiable, while imaging procedures may fall in the $30 \%$ of services that CMS estimates "do not improve patient care" [6]. Theoretically, the move to "bundled payments" for specific disease states or "episodes of care" as a part of a value-based healthcare model might make it easier to justify appropriately indicated imaging tests, which providers largely understand serve to advance overall optimal patient care.

\section{Mission Accomplished: Growth in Medical Imaging Has Been Flat for a Decade}

While the unfettered growth rates for medical imaging prior to 2010 served as reasonable justification for scrutiny and reform, continuing to invoke this historic pattern to justify additional interventions is not supported by current practice. According to the Medicare Payment Advisory Commission (MedPAC) Annual Reports to Congress, most recently published in March of 2020, utilization of medical imaging among Medicare beneficiaries has declined from 2010 to 2018 , while all other categories of medical services have continued to increase over the same timeframe [3] (see Fig. 1). Utilization data for echocardiography demonstrates a similar pattern. As outlined in various Medicare Payment Advisory (MedPAC) reports over the last 10 years, echocardiography procedures performed on Medicare beneficiaries nearly doubled between 2000 and 2009, before experiencing a sharp

\section{Growth in Volume of Services to Medicare} Beneficiaries, 2000 - 2018

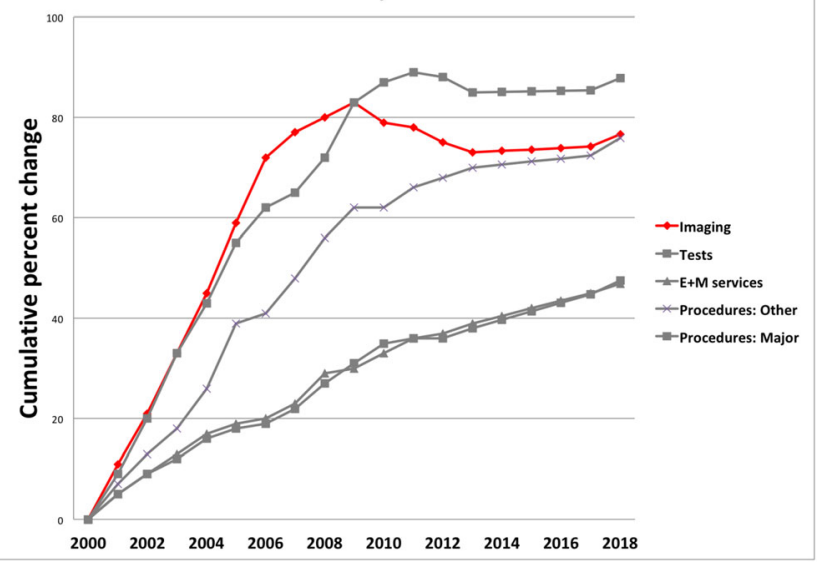

Fig. 1 2000-2018 growth of Medicare services for beneficiaries. Growth in medical imaging volume depicted in red (adapted from MedPac Report to Congress: 2019 and 2020 Medicare Payment Policy Update. http:// www.medpac.gov/)

correction with a $17 \%$ cumulative decline from 2009 to 2013. From 2013-2015, the last 2 years for which specific echocardiography data is reported, utilization has been essentially flat, decreasing $0.8 \%$ between 2013 and 2014, and increasing for the first time by $0.4 \%$ from 2014 to 2015 . From 2015 to 2018, combined "ultrasound" imaging procedures performed on Medicare beneficiaries has increased by $1.4 \%$ [3].

The reason for this imaging utilization plateau is undoubtedly multifactorial, with private payer prior authorization programs, declining reimbursements, and the intense focus on healthcare utilization in general all contributing. But the proactive development of Appropriate Use Criteria by physician specialty societies, and the message and guidance this sent to providers across the country, was assuredly among the most important reasons for the moderation in cardiac imaging utilization.

\section{Development of the AUC}

The initial AUC for echocardiography were released in 2007 (transthoracic echocardiography) and 2008 (stress echocardiography) and later revised, combined, and broadened in 2011 in an attempt to address the entirety of echocardiography practice [8-10]. Subsequent investigation has confirmed the 2011 AUC for echocardiography to be comprehensive, addressing $98 \%$ of indications in a consecutive cohort of over 2000 transthoracic, stress, and transesophageal echocardiograms [11]. All AUC documents initially categorized studies as "appropriate," "uncertain," and "inappropriate" [10]. The nomenclature has since been revised such that the AUC now categorize procedures as "appropriate," "may be appropriate," and 
"rarely appropriate." The nomenclature change was adopted in an attempt to acknowledge that there are nuances in medical care, including scenarios where AUC may not completely capture all clinical factors that contribute to a provider's decision to order a procedure or service. The primary purpose of this nomenclature change was to signal to payers that even "rarely appropriate" studies may contribute to optimal patient care, and thus may occasionally warrant reimbursement of a small number of studies in any practice setting.

It is important to note that AUC were developed as a supplement to, not a surrogate for, practice guidelines, as they have a very different purpose. Practice guidelines intend to direct a more rigid standard of care, while AUC attempt to identify clinical scenarios for which performance of an echocardiogram is generally reasonable and thus warrants reimbursement. Inherent in this purpose is that multiple competing modalities may potentially be reasonable in a particular clinical scenario, but that performing all of them may not. This issue has been acknowledged and addressed with the development of AUC to guide multimodality imaging.

The goal of multimodality AUC is not to determine a rank order of tests from most to least appropriate, but rather to "provide a comprehensive resource" for cardiac imaging selection for a particular disease category, which "encompasses multiple imaging modalities." [12]. These AUC aim to form a scenario-specific framework that can improve and streamline provider decision-making, but have inherent flexibility, such that the final decision remains at the discretion of the ordering practitioner [13]. Multimodality AUC have now been developed for the evaluation of valvular heart disease, cardiac structure and function, congenital heart disease, and stable ischemic heart disease $[14,15]$. In addition, AUC addressing particular clinical scenarios, such as cardiovascular imaging for the evaluation of patients with chest pain in the emergency

Table 1 Currently available ACC/AHA Appropriate Use Criteria addressing echocardiographic procedures (All AUC documents available at www.ACC.org)

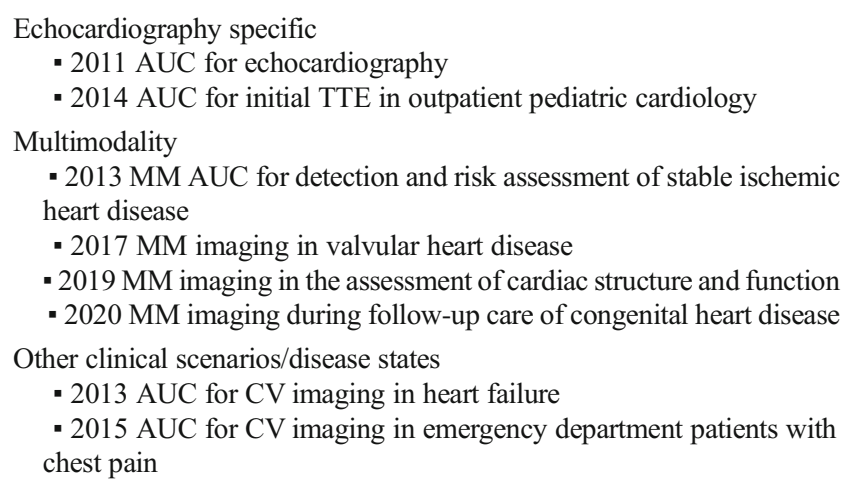

$M M$, multimodality; TTE, transthoracic echocardiography; $C V$, cardiovascular department, provide multimodality guidance [16] (see Table 1). Echocardiography procedures are featured prominently in each of these documents.

\section{Clinical Impact of the AUC}

Soon after its development, multiple implementation studies confirmed the feasibility of application of the AUC for echocardiography, and its ability to define and stratify clinical practice. In subsequent years, the impact of AUC on utilization has been reported. A large-scale meta-analysis found an improvement in rates of appropriate utilization as a result of the 2011 AUC, with TTE performing the best among multimodality imaging techniques studied [17].

The Echo WISELY Trial, published in 2017, was the first and largest prospective, multicenter, randomized controlled trial of an AUC-based intervention. This study sought to describe echo utilization and to investigate the effect of an AUCbased educational intervention on the TTE ordering habits of both primary care providers and cardiologists $[18 \bullet \bullet]$. The intervention arm received an educational video describing the AUC, instructions on how to download the ASE decision support app (available through Apple App Store ${ }^{\mathrm{TM}}$ ), as well as monthly feedback summarizing individual TTE ordering behavior, including frequency of "rarely appropriate" studies. While this did not change the rate of TTE ordering between groups (not powered to do so), the intervention group ordered significantly fewer "rarely appropriate" studies than the control group (8.6\% vs $11.1 \%)$ [18••].

The impact of "rarely appropriate" echocardiograms on patient care was found to go well beyond the imaging study itself. Sub-analysis of the Echo WISELY Trial has demonstrated that patients with congestive heart failure had fewer follow-up visits and had lower odds of receiving evidence-based medications (beta blockers) and interventions (implanted cardioverter defibrillators) when the cardiologist caring for them more frequently ordered "rarely appropriate" TTEs [19•]. Similar analysis has also been done on the subset of patients from the Echo WISELY Trial with coronary artery disease (CAD). Patients under the care of cardiologists who fell into the highest tertile of "rarely appropriate" echocardiogram-ordering were less likely to have cholesterol screening, hemoglobin A1c screening, or be prescribed a beta blocker or an aldosterone antagonist at 1 year when compared with the lower two tertiles of cardiologists. This was also associated with an increase in all-cause mortality at 1 year [20].

\section{Point of Order vs Point of Service}

The new CMS mandate for advanced imaging services requires implementation of the AUC through CDSMs at the 
point of test order [1]. Meanwhile, the only mandatory AUC tracking currently used in echocardiography practice are through ICAEL laboratory accreditation requirements at the location of test performance or point of service [4]. So, what if a similar future CMS mandate requires point of order AUC implementation for echocardiography?

Perhaps out of necessity, many point-of-order interventions have become more user-friendly, and several unique methods of incorporating AUC for echocardiography at the point of order have been developed. Eisman et al. developed automated systems for categorizing TTE indications according to the 2011 AUC for echocardiography, which have been validated against physician manual review. These systems were deemed equally efficacious while saving more than $45 \mathrm{~h}$ of physician review [21•]. The American Society of Echocardiography "Echo AUC App" is highly accessible, free, and allows rapid application of 98 Appropriate Use Criteria for echocardiography. Ordering providers enter desired echo modality and clinical indication details, and are provided immediate determination of appropriateness. A study of a precursor of the ASE Echo App found application in clinical care to be feasible and accurate [22]. More recent reports have found that the use of the ASE Echo App takes less than 20 s per echocardiogram, a critical metric in today's increasingly time-constrained clinical climate [23]. Finally, simplified applications of the AUC may be effective. Investigators in Australia developed a questionnaire based on 4 binary questions that successfully detected a high proportion of "rarely appropriate" TTEs without the need for the ordering or receiving provider to reference the detailed AUC [24].

As with the many ordering pathways and clinical algorithms providers already encounter embedded in EHRs, CDSMs to guide the ordering of cardiac imaging can be expected to promote guideline-driven patient care, aid practitioners less familiar with most recent evidence-based indications, and potentially reduce unnecessary testing and healthcare expenditures. While qualifying CDSMs for echocardiography will need to be refined and tested in the context of high volume clinical care, published experience to date regarding the echo AUC application at the point of order is promising.

\section{Rarely Appropriate Echocardiograms: Does This Mean Never Appropriate?}

Investigations in a variety of practice settings report that the vast majority of echocardiograms performed in clinical practice are found to be appropriate according to the AUC, but that in every practice setting "rarely appropriate" echocardiograms are also identified. As a result, common characteristics of "rarely appropriate" studies have been of great interest, as they might guide interventions aimed at limiting unnecessary echo procedures, optimizing clinical practice, and constraining costs (see Table 2). Two clear themes among "rarely appropriate" studies have emerged. First, repeat studies are much more likely to be "rarely appropriate" than initial echocardiograms $[11,25,26]$. This is not surprising given that the AUC provide clear guidance regarding the interval at which followup testing would be reasonable for a number of cardiac findings (e.g., a repeat echo to assess mild valvular disease would not be appropriate for at least 3 years, in the absence of a clinical change). This finding is not surprising to most ordering practitioners; however, many are not fully aware of specific recommendations for minimal intervals prior to repeat testing, or may not be aware of the date or even the presence of a prior echocardiogram. As a result, AUC implementation studies have found repeat echocardiograms to be the most fertile target for education and other programs aimed at the reduction of unnecessary echocardiograms.

The second theme relates to first time echocardiograms, with outpatient initial echocardiograms more commonly "rarely inappropriate" than inpatient initial echocardiograms $[11,25,26]$. This is also not unexpected given that most inpatients have symptoms or a "change in clinical status," a core feature establishing many AUC indications as "appropriate" [25-27]. Other characteristics such as practice setting (community vs academic) have not been shown to significantly affect the rate of "rarely appropriate" echocardiograms [25, 28 ], and physician's ordering specialty (cardiologist vs other) may or may not alter the rate of rarely appropriate testing, depending on the population studied $[8,11,25]$.

Table 2 Common "Rarely Appropriate" (formerly termed "Inappropriate") indications for which transthoracic echocardiograms are performed in clinical practice according to the 2011 AUC $[8,11$, $18 \bullet \bullet, 25]$

\footnotetext{
Initial TTE imaging

- Routine preoperative evaluation of ventricular function with no symptoms or signs of cardiovascular disease

- Routine evaluation of systemic hypertension without symptoms or signs of heart disease

- Lightheadedness/presyncope when there are no other symptoms or signs of cardiovascular disease

- Suspected endocarditis in a patient with transient fever without evidence of bacteremia or new murmur

Repeat (follow-up) TTE imaging

- Routine surveillance of ventricular function with known CAD and no change in clinical status or cardiac examination

- Routine surveillance of a prosthetic valve ( $<3$ years) if no known or suspected dysfunction

- Routine surveillance ( $<1$ year) of moderate to severe valvular stenosis without change in clinical status or cardiac exam

- Routine surveillance ( $<3$ year) of mild valvular stenosis without a change in clinical status or cardiac examination

- Routine surveillance $(<1$ year) of heart failure (systolic or diastolic) when there is no change in clinical status or cardiac examination
} 
It is important to note, however, that "rarely appropriate" does not mean "never appropriate." Clinical context is important, and it should be recognized that the process of AUC designation may occasionally be unable to fully capture all factors that contribute to echo ordering in real world clinical practice. Evidence supporting this conclusion is that "rarely appropriate" echocardiograms are found to have a not insignificant rate of echo abnormalities, ranging between 4 and $17 \%$ depending on the population studied [26, 29]. While these abnormalities may be incidental and cannot be used alone to justify a test, they do suggest that "rarely appropriate" echocardiograms may reveal important findings that guide clinical decision-making. Implicit in the methodology of the new CMS mandate appears to be an acknowledgment that a small rate of "rarely appropriate" imaging tests will be expected. The CMS process requires reporting AUC designation at the point of order, but does not initially intend to deny claims based on appropriateness designation. However, "outliers," presumably those with higher rates of "rarely appropriate" testing will be subject to prior authorization procedures [1].

\section{Correlation Between AUC and Echo Abnormalities}

It might be expected that echo abnormalities would represent an excellent metric to stratify appropriateness. Thus, logic would suggest that more abnormal echo findings would be seen on "appropriate" studies and fewer seen on "rarely appropriate" studies. This hypothesis has borne out in multiple prior investigations of the AUC for echocardiography. A subanalysis of the Echo WISELY Trial found that in an ambulatory population, $89.6 \%$ of identified abnormalities were found on "appropriate" scans as compared with only $4.3 \%$ on "inappropriate" scans [29]. Similarly, "appropriate" scans were more likely to reveal greater than one abnormality when compared with "rarely appropriate" scans. These echocardiographic abnormalities appear to have clinical relevance, as patients who had an "appropriate" TTE had a higher rate of hospitalization and composite of death and hospitalization [29]. Multiple other prior studies in diverse populations have similarly demonstrated the ability of the AUC to stratify echo abnormalities, with appropriate studies identifying many more echo abnormalities [23, 25, 30].

While echo abnormalities are one reasonable metric, it is important to note that they are not a perfect surrogate for appropriateness. Take for example a patient who is referred for an echocardiogram to evaluate for a cardiac cause of new onset dyspnea, a common and appropriate indication for echo according to the AUC. The echocardiogram may well be normal, which might prompt pulmonary testing for the cause of symptoms. This of course would meet every reasonable definition of appropriateness in clinical care.

\section{A Word on Underuse}

With the aggressive interventions and successful moderation of the utilization of echocardiography procedures over the last decade, concern about underutilization has arisen. While AUC implementation programs are aimed at unnecessary echo use, rigid and poorly designed prior authorization programs and across the board reimbursement cuts have served to suppress all echo utilization. Increasingly, practice settings and disease states in which echocardiograms could advance optimal patient care but are not being performed are being identified. For example, Papolos et al. queried the Nationwide Inpatient Sample (NIS) database and determined the most frequent clinical scenarios for which echocardiography is ordered [31]. The investigators found that patients hospitalized with myocardial infarction, heart failure, cardiac dysrhythmia, acute cerebrovascular ischemia, or sepsis who underwent echo faced a lower likelihood of inpatient mortality than did patients without echo. It was found that echo was used in only $8 \%$ of approximately 3.7 million cases with these 5 diagnoses [31]. Undoubtedly, a majority of these aforementioned indications would fall into the "appropriate" category according to the AUC, and practice guidelines provide specific support for echocardiography for many of them, as well. These findings highlight that AUC implementation and education should not only focus on limiting "rarely appropriate" studies, but also recognizing clinical scenarios in which echocardiography is both "appropriate" and may advance optimal clinical care.

\section{Conclusion}

Echocardiography remains the most widely utilized cardiac imaging modality and is established as a mainstay of patient care. Excessive growth rates early in the millennium focused scrutiny on all cardiac imaging modalities, including echocardiography, but utilization has now moderated over last decade. The development of Appropriate Use Criteria has been a major contributor to this change in practice. However, the effect of the AUC for echocardiography to date has been largely indirect, serving to educate and focus echo providers on appropriate utilization, without widespread direct incorporation of the AUC at the point of order or as part of prior authorization programs. This result was the ideal goal of the AUC when developed, to spur optimization of practice and thus stave off more intrusive interventions. This initial mission has been accomplished by the AUC for echocardiography. However, other cardiac imaging modalities have not been as lucky, and now, a new CMS mandate will require point of order application of AUC for these "advanced" imaging studies as of January 1, 2021, to be eligible for reimbursement. The experience with full implementation of this mandate will go a long 
way toward determining the future for echocardiography. If it is seamless, there may be little reason not to issue a future mandate for AUC implementation for echo procedures. While this prospect was once considered the worst-case scenario, over a decade of experience and familiarity with the AUC now suggests that the practice of echocardiography will not only adapt but also continue to thrive.

\section{Compliance with Ethical Standards}

Conflict of Interest Ian Hackett and R. Parker Ward declare that they have no conflict of interest.

Human and Animal Rights and Informed Consent This article does not contain any studies with human or animal subjects performed by any of the authors.

\section{References}

Papers of particular interest, published recently, have been highlighted as:

- Of importance

•. Of major importance

1. Appropriate Use Criteria Program. www.cms.gov. Online. Accessed March 30, 2020.

2. Byrd BF 3rd, Abraham TP, Buxton DB, et al. A summary of the American Society of Echocardiography Foundation value-based healthcare: summit 2014: the role of cardiovascular ultrasound in the new paradigm. J Am Soc Echocardiogr. 2015;28:755-69.

3. MEDPAC. Report to the congress: Medicare payment policy. Washington DM, 2000-2020.

4. Commission-Echocardiography IA. IAC Standards and Guidelines for Adult Echocardiography Accreditation. Online, 2018.

5. Echocardiography ASo. ASE update payer policies: July 2013. 2013.

6. NHE Fact Sheet. In: Services CfMaM, editor, 2019.

7. Douglas PS, Cerqueira MD, Berman DS, Chinnaiyan K, Cohen MS, Lundbye JB, et al. The future of cardiac imaging: report of a think tank convened by the American College of Cardiology. JACC Cardiovasc Imaging. 2016;9:1211-23.

8. Ballo P, Bandini F, Capecchi I, Chiodi L, Ferro G, Fortini A, et al. Application of 2011 American College of Cardiology Foundation/ American Society of Echocardiography appropriateness use criteria in hospitalized patients referred for transthoracic echocardiography in a community setting. J Am Soc Echocardiogr. 2012;25:589-98.

9. Douglas PS, Khandheria B, Stainback RF, et al. ACCF/ASE/ ACEP/ASNC/SCAI/SCCT/SCMR 2007 appropriateness criteria for transthoracic and transesophageal echocardiography: a report of the American College of Cardiology Foundation Quality Strategic Directions Committee Appropriateness Criteria Working Group, American Society of Echocardiography, American College of Emergency Physicians, American Society of Nuclear Cardiology, Society for Cardiovascular Angiography and Interventions, Society of Cardiovascular Computed Tomography, and the Society for Cardiovascular Magnetic Resonance endorsed by the American College of Chest Physicians and the Society of Critical Care Medicine. J Am Coll Cardiol. 2007;50:187-204.
10. Lindekleiv H, Lochen ML, Mathiesen EB, Njolstad I, Wilsgaard T, Schirmer H. Echocardiographic screening of the general population and long-term survival: a randomized clinical study. JAMA Intern Med. 2013;173:1592-8.

11. Mansour IN, Razi RR, Bhave NM, Ward RP. Comparison of the updated 2011 appropriate use criteria for echocardiography to the original criteria for transthoracic, transesophageal, and stress echocardiography. J Am Soc Echocardiogr. 2012;25:1153-61.

12. Mastos J, Strom JB. An Executive Summary of the 2019 AUC for multimodality imaging in the assessment of cardiac structure and function in Nonvalvular heart disease. 2019. www.acc.org/latest-incardiology/articles/.

13. Doherty JU, Kort S, Mehran R, et al. ACC/AATS/AHA/ASE/ ASNC/HRS/SCAI/SCCT/SCMR/STS 2019 appropriate use criteria for multimodality imaging in the assessment of cardiac structure and function in nonvalvular heart disease: a report of the American College of Cardiology Appropriate Use Criteria Task Force, American Association for Thoracic Surgery, American Heart Association, American Society of Echocardiography, American Society of Nuclear Cardiology, Heart Rhythm Society, Society for Cardiovascular Angiography and Interventions, Society of Cardiovascular Computed Tomography, Society for Cardiovascular Magnetic Resonance, and the Society of Thoracic Surgeons. J Am Coll Cardiol. 2019;73:488-516.

14. Doherty JU, Kort S, Mehran R, Schoenhagen P, Soman P. ACC/ AATS/AHA/ASE/ASNC/HRS/SCAI/SCCT/SCMR/STS 2017 appropriate use criteria for multimodality imaging in valvular heart disease: a report of the American College of Cardiology Appropriate Use Criteria Task Force, American Association for Thoracic Surgery, American Heart Association, American Society of Echocardiography, American Society of Nuclear Cardiology, Heart Rhythm Society, Society for Cardiovascular Angiography and Interventions, Society of Cardiovascular Computed Tomography, Society for Cardiovascular Magnetic Resonance, and Society of Thoracic Surgeons. J Am Coll Cardiol. 2017;70: 1647-72.

15. Sachdeva R, Valente AM, Armstrong AK, et al. ACC/AHA/ASE/ $\mathrm{HRS} / \mathrm{ISACHD} / \mathrm{SCAI} / \mathrm{SCCT} / \mathrm{SCMR} / \mathrm{SOPE} 2020$ appropriate use criteria for multimodality imaging during the follow-up care of patients with congenital heart disease: a report of the American College of Cardiology Solution Set Oversight Committee and Appropriate Use Criteria Task Force, American Heart Association, American Society of Echocardiography, Heart Rhythm Society, International Society for Adult Congenital Heart Disease, Society for Cardiovascular Angiography and Interventions, Society of Cardiovascular Computed Tomography, Society for Cardiovascular Magnetic Resonance, and Society of Pediatric Echocardiography. J Am Coll Cardiol. 2020;75:657-703.

16. Rybicki FJ, Udelson JE, Peacock WF, et al. 2015 ACR/ACC/AHA/ AATS/ACEP/ASNC/NASCI/SAEM/SCCT/SCMR/SCPC/ SNMMI/STR/STS appropriate utilization of cardiovascular imaging in emergency department patients with chest pain: a joint document of the American College of Radiology Appropriateness Criteria Committee and the American College of Cardiology Appropriate Use Criteria Task Force. J Am Coll Cardiol. 2016;67: 853-79.

17. Fonseca R, Negishi K, Otahal P, Marwick TH. Temporal changes in appropriateness of cardiac imaging. J Am Coll Cardiol. 2015;65: 763-73.

18.• Bhatia RS, Ivers NM, Yin XC, et al. Improving the appropriate use of transthoracic echocardiography: the Echo WISELY Trial. J Am Coll Cardiol. 2017;70:1135-44 Findings of this study demonstrate that AUC-based education and feedback intervention reduces the number of "rarely appropriate" TTEs ordered by physicians in a variety of ambulatory care environments. 
19. Tharmaratnam T, Bouck Z, Sivaswamy A, et al. Association between physicians' appropriate use of echocardiography and subsequent healthcare use and outcomes in patients with heart failure. $\mathrm{J}$ Am Heart Assoc. 2020;9:e13360 Patients of cardiologists who more frequently ordered "rarely appropriate" TTEs had lower odds of receiving cardiovascular risk factor assessment and prescription of appropriate heart failure guideline-directed medical therapy.

20. Tharmaratnam T, Bouck Z, Sivaswamy A, et al. Low-value transthoracic echocardiography, healthcare utilization, and clinical outcomes in patients with coronary artery disease. Circ Cardiovasc Qual Outcomes. 2019;12:e006123.

21. Eisman AS, Weiner RB, Chen ES, et al. An automated system for categorizing transthoracic echocardiography indications according to the echocardiography appropriate use criteria. AMIA Annu Symp Proc. 2017;2017:670-8 This study demonstrates both the practicality and accessibility of using computer models to determine appropriateness of an echocardiogram at the point of order.

22. Bhave NM, Mansour IN, Veronesi F, Razi RR, Lang RM, Ward RP. Use of a web-based application of the American College of Cardiology Foundation/American Society of Echocardiography appropriateness use criteria for transthoracic echocardiography: a pilot study. J Am Soc Echocardiogr. 2011;24:271-6.

23. Obeng-Okyere P, Norbu T, Hahn H. Point of care use of an appropriate use criteria smartphone app for echocardiography. J Am Soc Echocardiogr. 2019;32:1048-9.

24. Fonseca R, Pathan F, Marwick TH. Development and validation of a screening tool for the identification of inappropriate transthoracic echocardiograms. BMJ Open. 2016;6:e012702.

25. Bhatia RS, Carne DM, Picard MH, Weiner RB. Comparison of the 2007 and 2011 appropriate use criteria for transthoracic echocardiography in various clinical settings. J Am Soc Echocardiogr. 2012;25:1162-9.

26. Ward RP, Mansour IN, Lemieux N, Gera N, Mehta R, Lang RM. Prospective evaluation of the clinical application of the American College of Cardiology Foundation/American Society of
Echocardiography Appropriateness Criteria for transthoracic echocardiography. JACC Cardiovasc Imaging. 2008;1:663-71.

27. Douglas PS, Garcia MJ, Haines DE, et al. ACCF/ASE/AHA/ ASNC/HFSA/HRS/SCAI/SCCM/SCCT/SCMR 2011 appropriate use criteria for echocardiography. a report of the American College of Cardiology Foundation Appropriate Use Criteria Task Force, American Society of Echocardiography, American Heart Association, American Society of Nuclear Cardiology, Heart Failure Society of America, Heart Rhythm Society, Society for Cardiovascular Angiography and Interventions, Society of Critical Care Medicine, Society of Cardiovascular Computed Tomography, Society for Cardiovascular Magnetic Resonance American College of Chest Physicians. J Am Soc Echocardiogr. 2011;24:229-67.

28. Ward RP, Krauss D, Mansour IN, Lemieux N, Gera N, Lang RM. Comparison of the clinical application of the American College of Cardiology Foundation/American Society of Echocardiography Appropriateness Criteria for outpatient transthoracic echocardiography in academic and community practice settings. J Am Soc Echocardiogr. 2009;22:1375-81.

29. Law TK, Bouck Z, Yin XC, Dudzinski D, Thavendiranathan P, Nesbitt GC, et al. Association between TTE appropriateness, echocardiographic findings, and clinical outcomes. JACC Cardiovasc Imaging. 2019;12:379-81.

30. McClelland ILR, Mor-Avi V, Ward RP. Prevalence of clinically important abnormalities found on TTEs ordered for indication of heart murmur found on physical examination. J Am Soc Echocardiogr. 2020;S0894-7317(20):30098-5. https://doi.org/10. 1016/j.echo.2020.02.010.

31. Papolos A, Narula J, Bavishi C, Chaudhry FA, Sengupta PP. U.S. hospital use of echocardiography: insights from the nationwide inpatient sample. J Am Coll Cardiol. 2016;67:502-11.

Publisher's Note Springer Nature remains neutral with regard to jurisdictional claims in published maps and institutional affiliations. 